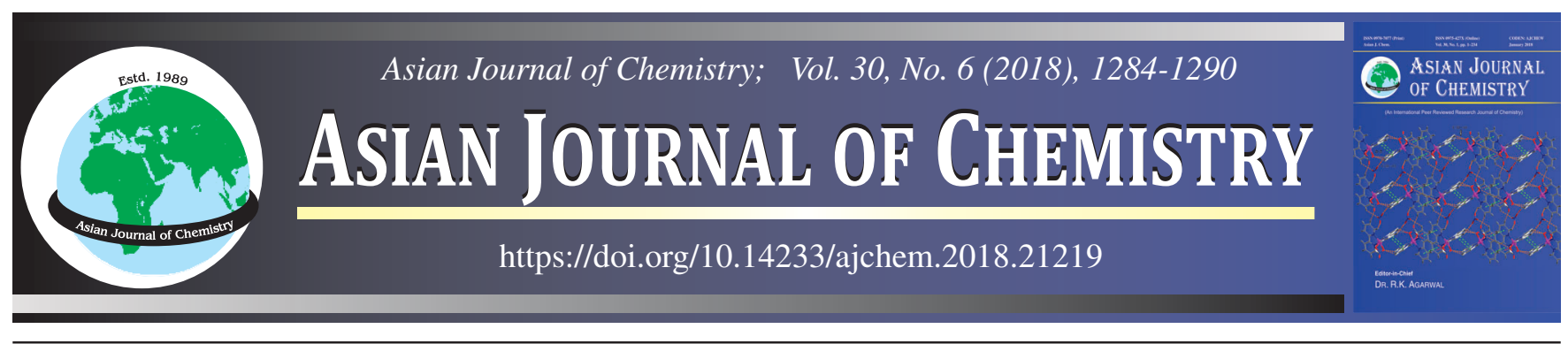

\title{
Facile Synthesis of Graphene Oxide-Nano Titania Composites and Evaluation for Visible Light Assisted Photocatalytic Degradation of Rhodamine B
}

\author{
Soma SeKhar Ryali ${ }^{1,2, *}$ and Paul Douglas SANASI ${ }^{3}$
}

${ }^{1}$ Department of Chemistry, Anil Neerukonda Institute of Technology \& Sciences (A), Visakhapatnam-531 162, India

${ }^{2}$ Department of Chemistry, Jawaharlal Nehru Technological University, Kakinada-533 003, India

${ }^{3}$ Department of Engineering Chemistry, AU College of Engineering (A), Andhra University, Visakhapatnam-530 003, India

*Corresponding author: E-mail: somasekhar.chemistry@anits.edu.in

Received: 10 January 2018;

Accepted: 3 March 2018;

Published online: 30 April 2018;

AJC-18881

Nano titania composite materials exfoliated with varying weight percentage of graphene oxide were synthesized and evaluated for visible light assisted photocatalytic degradation of rhodamine-B. Titanium tetrachloride was hydrolyzed in water medium under organic solvent free conditions followed by ultrasonic addition of the as prepared $1 \%$ aqueous solution of graphene oxide. The morphology and structural properties of the composite materials $(1,2,5,10,15$ wt \% graphene oxide-nano titania) prepared has been characterized using X-ray diffraction (XRD), Fourier transform infrared spectroscopy (FTIR), UV-visible diffuse reflectance spectra (UV-Vis DRS), high resolution transmission electron microscope (HRTEM), field emission-scanning electron microscopy (FESEM) and UV-visible spectroscopy. A superior activity was observed with $10 \mathrm{mg}$ of $10 \%$ graphene oxide-nano titania composite material. The $100 \%$ degradation of rhodamine-

$\mathrm{B}$ was observed in $60 \mathrm{~min}$ and other optimum conditions were established.

Keywords: Graphene Oxide, Nano titania, Photocatalytic activity, Rhodamine-B.

\section{INTRODUCTION}

Visible light induced photocatalytic degradation of organic pollutants present in industrial wastewater is a well known and established technique in the area of green chemistry to purify wastewater [1]. Anatase form of titania is the most thoroughly investigated material in the literature and seems to be a promising photocatalyst for the detoxification of organic pollutants [2-4]. Owing to its low toxicity, high photostability and high photo-efficiency it acts as a unique photocatalyst. However, there are few snagging issues with this material due to rapid electron-hole recombination in $10^{-9} \mathrm{~s}$ which results in low quantum efficiency, a wide band gap of $3.2 \mathrm{eV}$ limiting its usage mostly in the UV region [5]. Developing a heterogeneous photocatalyst to bring the usage of titania particles in the visible region is a conventional route to enhance the photocatalytic degradation of organic dyes like anionic doping with $\mathrm{C}, \mathrm{N}$ and $\mathrm{S}$ ions or transition metal ions or formation of nano-composites with suitable materials to yield high surface to volume ratio $[6,7]$. Efforts have been made to synthesize titania-gold nano composites, CdS/CdSe-titania hybrids, etc. for inhibition of electronhole recombination and extending the photocatalytic process in visible region [8]. Modifying titania with a nano-structured carbonaceous substance like graphene oxide (GO) has been gaining importance to contrive visible-light responsive materials.

Graphene oxide is a two dimensional carbon material synthesized from graphite and possess high thermal and electrical conductivity. The $s p^{2}$ hybridized carbon atoms within the material are highly capable in storing and shuttling the electrons [9]. A large number of surface property enhancing oxygen-containing groups on both sides of a single graphite sheet overcomes the inter sheet van der Waals force and enlarges the interlayer spacing [10]. The sheets in such an expanded structure can be easily pulled up using an external force by ultrasonication. This technique would not lead to any dissociation of the sheets, due to its strong coupling leading to an advanced hybrid materials for various applications including photocatalysis.

In many works, nano-structured titania catalysts have been synthesized by hydrolysis of titanium isopropoxide and titanium tert-butoxide as the precursor materials [11]. Samples derived from organic solvent free controlled hydrolysis of titanium tetrachloride were reported to be highly photoactive and the final yield does not give rise to the formation of organic impurities [12] . 1:50 [precursor]/[solvent] volume ratio of $\mathrm{TiCl}_{4} / \mathrm{H}_{2} \mathrm{O}$ was reported as a unique combination for synthesis of anatase nano titania material having best crystallinity, enhanced photocatalytic activity and high surface area [11]. 
In earlier works, photocatalytic activity of Degussa P25/GO composites was performed $[5,13,14]$. Hydrothermally prepared $\mathrm{TiO}_{2} / \mathrm{GO}$ sheets and their photocatalytic activity for hydrogen evolution from water was also reported [6]. Unlike these reports, the present work reports synthesis of a novel combination of graphene oxide-nano titania composite materials by one step organic solvent free controlled hydrolysis of $\mathrm{TiCl}_{4}$ by maintaining 1:50 volume ratio of $\mathrm{TiCl}_{4} / \mathrm{H}_{2} \mathrm{O}$ in the dispersion of aqueous solution of graphene oxide under ultrasonication. It was noticed that the materials were responsive in visible light region and enhanced photocatalytic performance was observed with increase in wt $\%$ of graphene oxide along with high surface area of nano titania in the composite materials. These composites have been characterized using XRD, FESEM, HRTEM-SAED (selected area electron diffraction), FTIR and UV-Vis DRS. It has been medically proven that contaminated drinking water with rhodamine dye could lead to subcutaneous tissue borne sarcoma which is highly carcinogenic [15]. Hence, rhodamine B dye was selected as the probe molecule for the photocatalysis study under visible light in the presence of prepared composite materials.

\section{EXPERIMENTAL}

Titanium tetrachloride $\left(\mathrm{TiCl}_{4}\right)$, graphite powder, potassium permanganate, phosphoric acid, sulphuric acid, hydrogen peroxide were procured with AR grade quality from SD-Fine analytical grade of $99 \%$ purity. Rhodamine B (Finar), was selected as the probe molecule for photocatalytic degradation study under visible light whose chemical formula is $\mathrm{C}_{28} \mathrm{H}_{31} \mathrm{~N}_{2} \mathrm{O}_{3} \mathrm{Cl}$. The experimental solutions of the dye were prepared using distilled water.

The resulting composite materials were characterized using X-ray diffractometer (PANanalytical-X' Pert PRO, Japan) at room temperature using nickel filter $\mathrm{Cu}-\mathrm{K} \alpha$ radiation $(\lambda=$ $1.54059 \AA$ ) over wide range of $10^{\circ} \leq 2 \theta \leq 80^{\circ}$ with a scanning speed of $2 \mathrm{~min}^{-1}$. UV-visible diffuse reflectance spectra were recorded using single monochromator UV-2600 (optional ISR2600Plus, $\lambda$ upto $1400 \mathrm{~nm}$ ). The morphology of as-synthesized samples was investigated by field emission scanning electron microscopy (FESEM, LEO1550) and high resolution transmission electron microscopy (HRTEM, Joel/JEM 2100 model, source-LaB6). Fourier transform infrared spectral (FTIR) data was recorded from BRUKER ALPHA FT-IR with Opus 6.1 using $\mathrm{KBr}$ pellets at $4500-400 \mathrm{~cm}^{-1}$ region.

General procedure of in situ synthesis of $x \%$ GO-nano titania composites: Graphene oxide (GO) was prepared by well-known modified Hummers method [16] without any further modification. A standard solution of GO was prepared by dispersing $1 \mathrm{~g}$ of GO powder in $100 \mathrm{~mL}$ of distilled water and kept under ultrasonication. From this standard solution, $5 \mathrm{~mL}(0.05 \mathrm{~g})$, $10 \mathrm{~mL}(0.1 \mathrm{~g}), 25 \mathrm{~mL}(0.25 \mathrm{~g}), 50 \mathrm{~mL}(0.5 \mathrm{~g})$ and $75 \mathrm{~mL}(0.75 \mathrm{~g})$ of GO solution was dispersed in 5 separate beakers each containing $350 \mathrm{~mL}$ of distilled water under ultrasonication for a better distribution of GO particles. In order to maintain 1:50 $\mathrm{mL}$ ratio of $\mathrm{TiCl}_{4} / \mathrm{H}_{2} \mathrm{O}, 6.9 \mathrm{~mL}$ of $\mathrm{TiCl}_{4}$ was slowly added to each beaker containing $350 \mathrm{~mL}$ of distilled water and left for 30 min under ultrasonication to allow the exfoliation of GO particles on the surface of nanotitania particles. This [precursor]/ [solvent] volume ratio of $\mathrm{TiCl}_{4} / \mathrm{H}_{2} \mathrm{O}$ produces around $5 \mathrm{~g}$ of nano titania particles. The final mixtures were heated at around 100 to $120^{\circ} \mathrm{C}$ to vapourize chloride ions and the gel obtained was dried in an electrical hot air oven at $80^{\circ} \mathrm{C}$. In order to achieve anatase crystal phase in the nano titania particles, the composite materials were calcined at $400^{\circ} \mathrm{C}$ for $2 \mathrm{~h}$. The initial composite material (i.e., graphene oxide-nanotitania) was designated as $1 \% \mathrm{GO}-\mathrm{NT}$ (0.05 g of GO in $5.0 \mathrm{~g}$ of nano titania). Similarly, the other composite materials were designated as $2 \%$ GO-NT, $5 \%$ GO-NT, $10 \%$ GO-NT and $15 \%$ GO-NT, respectively.

Photocatalytic measurements: The photocatalytic activities of the composites in the degradation of rhodamine B dye solution were assessed by using a UV-visible spectrophotometer (Systronics-105, wavelength range: 340-960 nm). A known weight of the composite material was added to $100 \mathrm{~mL}$ of the wide range of concentrated dye solutions of rhodamine $\mathrm{B}\left(\lambda_{\max }=\right.$ $554 \mathrm{~nm}$ ) under continuous stirring. The suspensions were magnetically stirred for $30 \mathrm{~min}$ in dark to establish desorption/adsorption equilibrium. The photocatalytic study was carried out by placing the dye solutions under 400 watt metal halide lamp equipped with a wooden breakfront and an electric supply. Aliquots (5 $\mathrm{mL}$ ) were drawn under regular intervals of time, centrifuged and the translucent dye solution was analyzed by using UV-visible spectrophotometer. Percentage degradation of the dye was calculated using the following formula:

$$
\text { Photocatalytic degradation }(\%)=\left(\frac{\mathrm{C}_{\mathrm{o}}-\mathrm{C}_{\mathrm{t}}}{\mathrm{C}_{\mathrm{o}}}\right) \times 100
$$

where $\mathrm{C}_{\mathrm{o}}$ is the initial concentration of dye and $\mathrm{C}_{\mathrm{t}}$ is the concentration of dye at time t.

\section{RESULTS AND DISCUSSION}

X-ray diffraction analysis: The X-ray diffraction patterns of the synthesized composite materials were recorded in the $2 \theta$ range of $10^{\circ}$ to $80^{\circ}$ at a step interval of $0.02^{\circ}$ with the counting time of $5 \mathrm{~s}$ at each point. The XRD spectrum of graphene oxide (Fig. 1) shows a distinct peak at $2 \theta=10.2^{\circ}$ corresponding to the diffraction pattern $\mathrm{C}\left(\begin{array}{ll}0 & 0\end{array}\right)$. Fig. 2 shows the XRD patterns of nano titania and $\mathrm{x} \%$ GO-nano titania $(\mathrm{x}=1,2,5,10,15)$ at $2 \theta=25.25^{\circ}, 37.8^{\circ}, 54.5^{\circ}, 48.0^{\circ}$ for the diffraction patterns $\left(\begin{array}{ll}1 & 0\end{array}\right)$,

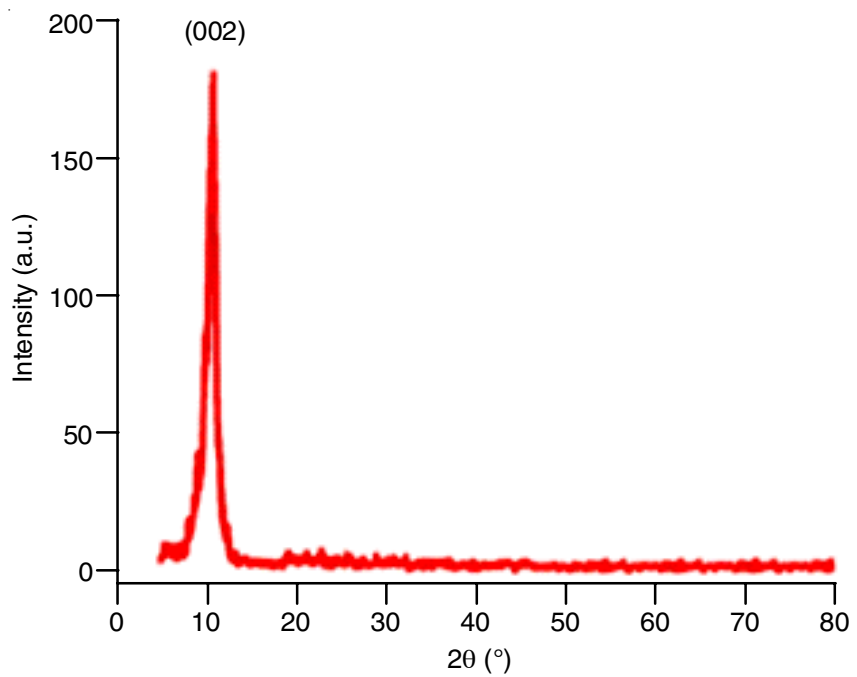

Fig. 1. XRD of graphene oxide 


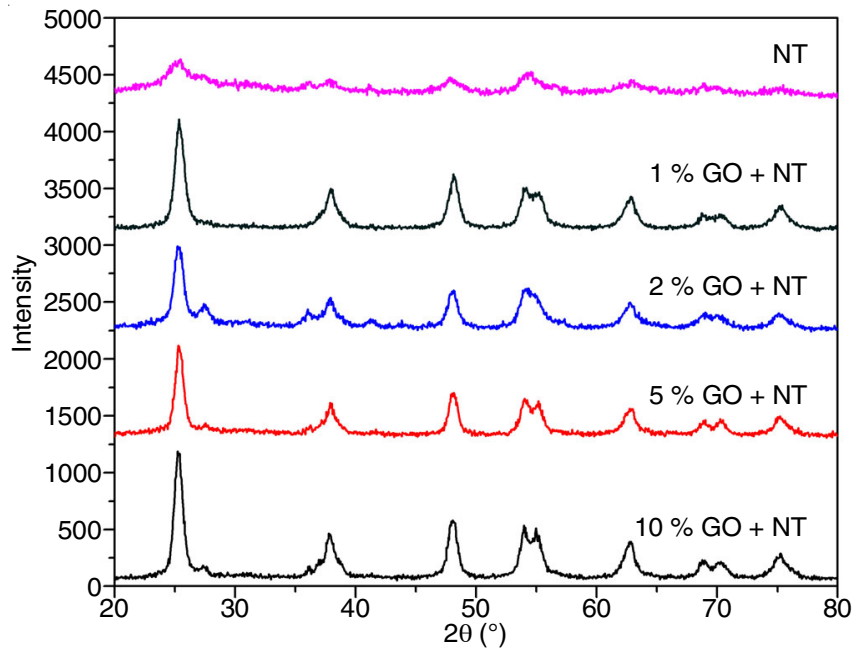

Fig. 2. XRD peaks of nano titania \& $x \%$ GO-NT composites

(0 0 4), (2 11 1) and (1 0 5), respectively indexing the dominant anatase phase (JCPDS 21-1272). All the diffraction peaks were in close agreement with the characteristic peaks of materials. The typical diffraction peak of graphene oxide was not observed in the XRD patterns of $x \%$ GO-nano titania composites and it may be due to well exfoliation of GO particles on the surface of nano titania particles or its low diffraction intensity. The peak broadening can be attributed to high crystalline nature and low particle size of the composite materials [11].

FTIR studies: Fig. 3 shows the FTIR spectra of graphene oxide particles. A broad peak at $3434.59 \mathrm{~cm}^{-1}$ indicates the presence of surface -OH stretching vibration. A small peak at $1726 \mathrm{~cm}^{-1}$ represents the $\mathrm{C}=\mathrm{O}$ stretching vibration of carboxylic groups at the edges of graphene oxide nano-sheets. The presence of absorption peaks at $1627.64 \mathrm{~cm}^{-1}$ was due to $\mathrm{O}-\mathrm{H}$ bending and aromatic $\mathrm{C}=\mathrm{C}$ stretching vibrations. Similarly, peaks at $1387.41 \mathrm{~cm}^{-1}$ was due to tertiary $\mathrm{C}-\mathrm{OH}$ stretching vibration and the results were in close agreement with the standard reports [5]. Fig. 4 shows the FTIR spectra of $x \%$ GO-nano titania composite materials. A peak at $3360 \mathrm{~cm}^{-1}$ represents the -OH stretching vibration of GO particles. An absorption peak at $1084 \mathrm{~cm}^{-1}$ corresponding to $\mathrm{C}-\mathrm{O}$ stretching mode of hydroxyl groups in graphene oxide was absent in $\mathrm{x} \% \mathrm{GO}$-nano titania composites. Similarly, absorption peaks in the range of $1000-400 \mathrm{~cm}^{-1}$ corresponding to Ti-O-Ti bonds shifted towards lower wavelength on incorporating graphene oxide. This can be attributed to the presence of both Ti-O-Ti and Ti-O-C bonds

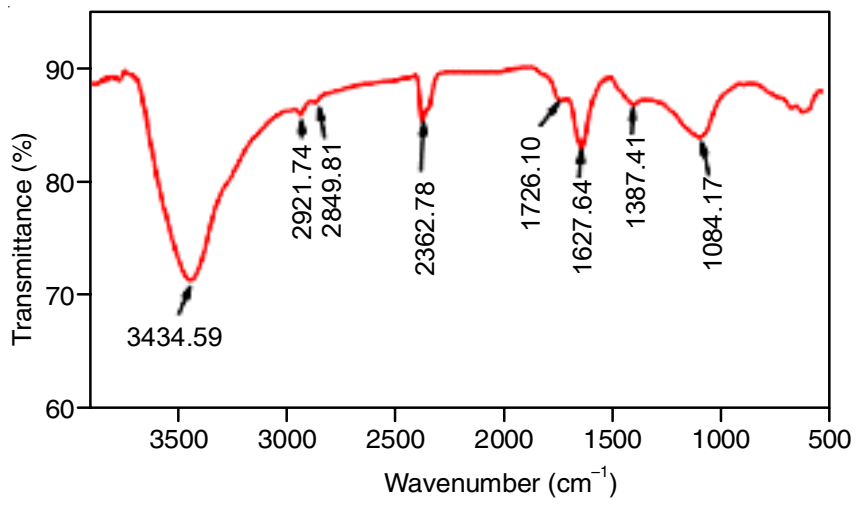

Fig. 3. FTIR spectra of graphene oxide

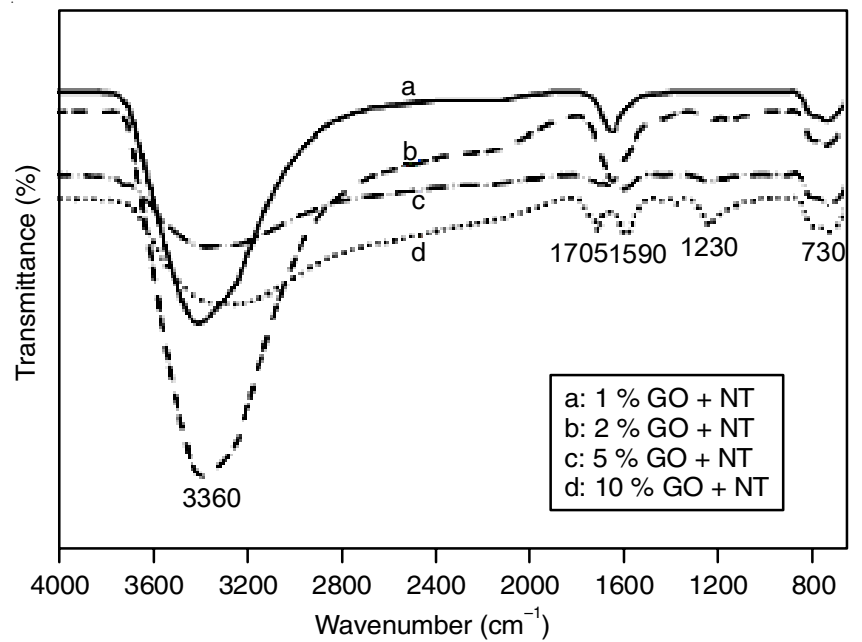

Fig. 4. FTIR peaks of $x \%$ GO-nano titania composites

in the composites indicating a strong chemical interaction between surface hydroxyl groups of nano titania and oxygen moieties in graphene oxide particles [5]. These results were almost in close agreement with the earlier works [13].

Morphology studies: The morphology and surface properties of the composite materials were recorded using electron microscopic instruments. Fig. 5 shows FESEM image of $10 \%$ GO-nano titania material. Spherical shaped particles were observed in the FESEM image (at $10 \mathrm{~nm}$ scale) tended to large aggregates. These results were almost similar to the reports of Zhang et al. [17]. Fig. 6a shows HRTEM image (at $2 \mathrm{~nm}$ scale) of $10 \%$ GO-nano titania composite material. It can observed that a perfect crystalline was formed in the nano titania particles with major (1 $\left.\begin{array}{lll}1 & 0\end{array}\right)$ anatase diffraction pattern and graphene oxide particles deposited on its surface. Fig. 6b clearly represents in the SAED image of $10 \%$ GO-nano titania (1 01 ) diffraction pattern of the nano titania particles. The results were very close to the reported works $[13,18]$.

UV-visible diffuse reflectance spectral studies: Fig. 7 shows the optical properties of the composite materials studied using UV-visible diffuse reflectance spectra of $\mathrm{x} \% \mathrm{GO}$-nano titania composite materials. It was clearly observed that the absorbance was increased with increase in wt \% of GO in the

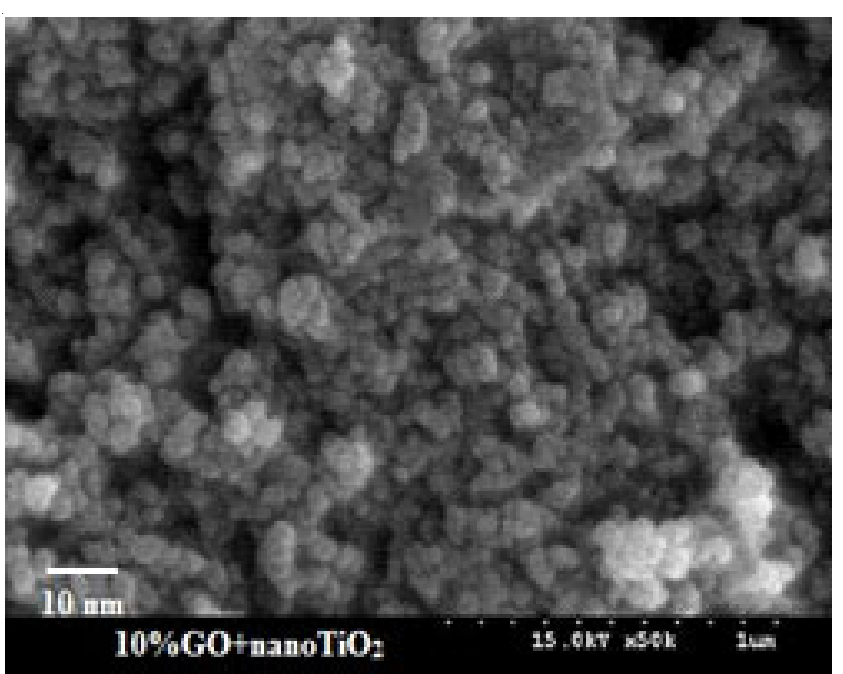

Fig. 5. FESEM image of $10 \%$ GO-nano titania composite material 


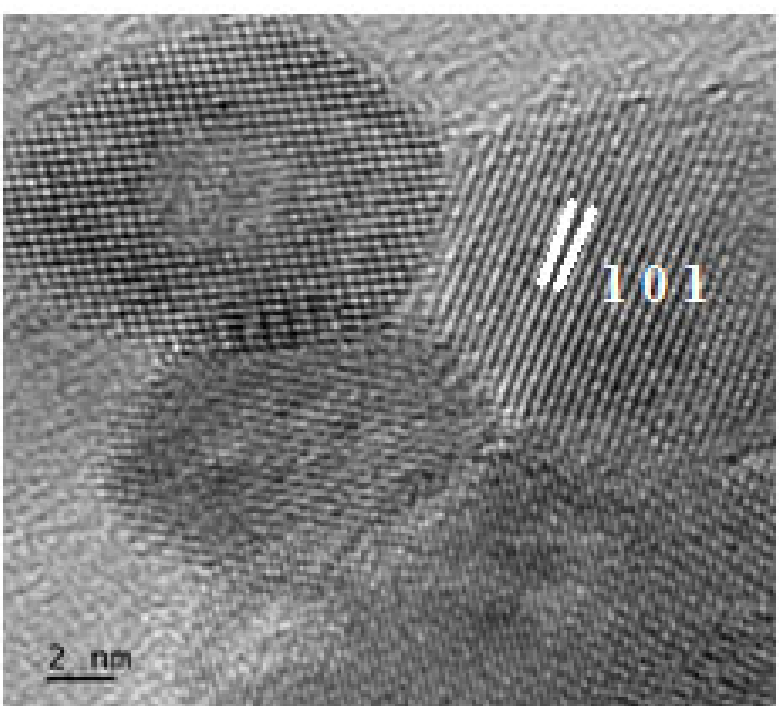

Fig. 6a. HRTEM image of $10 \%$ graphene oxide

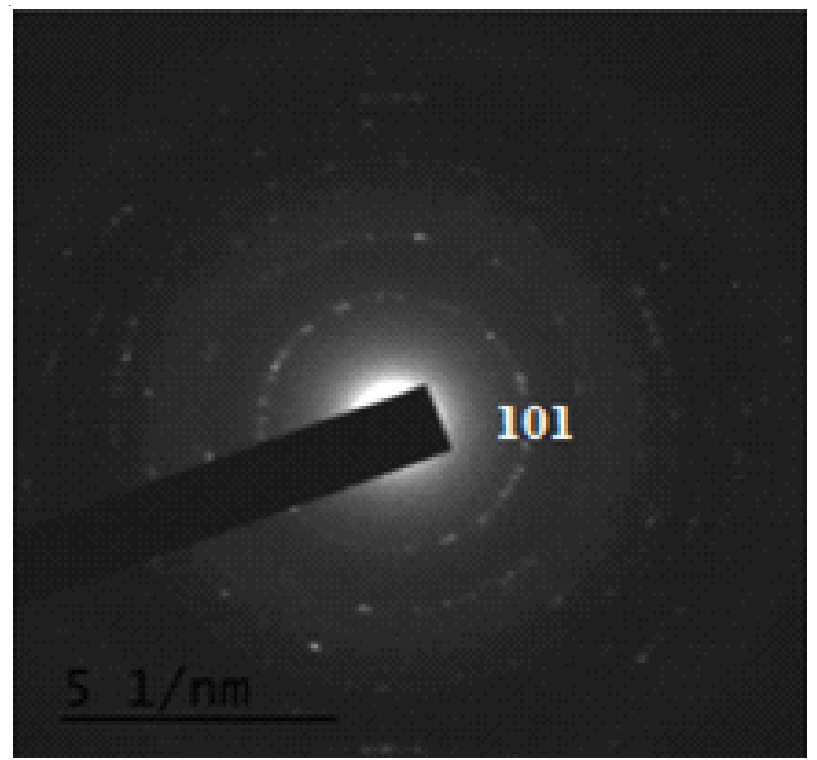

Fig. 6b. SAED pattern of $10 \%$ GO-nano titania composite material nano titania composite material

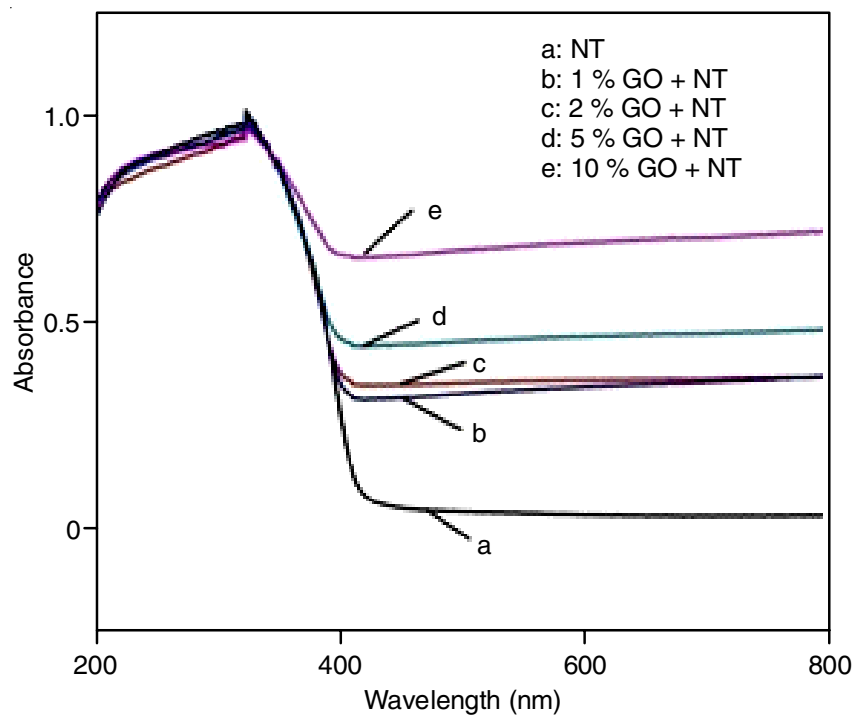

Fig. 7. UV-visible DRS spectra of the composites materials. This may be attributed to the already existing high surface to pore volume created by nano titania particles prepared by 1:50 mL of $\mathrm{TiCl}_{4} / \mathrm{H}_{2} \mathrm{O}$ and some restoration of $\pi$-conjugated system in graphene oxide particles. The shifting of absorption to visible region is also due to high visible absorption properties of GO relative to nano titania [13]. Enhanced visible light absorption indicates a strong chemical interaction between titanium ions and oxygen moieties in graphene oxide particles. These results were in close agreement with reported works $[1,6]$.

UV-visible spectroscopic studies: The UV-visible spectra for rhodamine-B (in aqueous medium) was recorded and a high intensity peak was observed at absorption wavelength, $\lambda_{\max }=554 \mathrm{~nm}$ by scanning the instrument in visible range. The percentage of degradation of rhodamine B was examined at various time intervals. Fig. 8 shows the absorption spectrum of rhodamine $\mathrm{B}$ in aqueous medium for $10 \mathrm{ppm}$ concentration of dye solution.

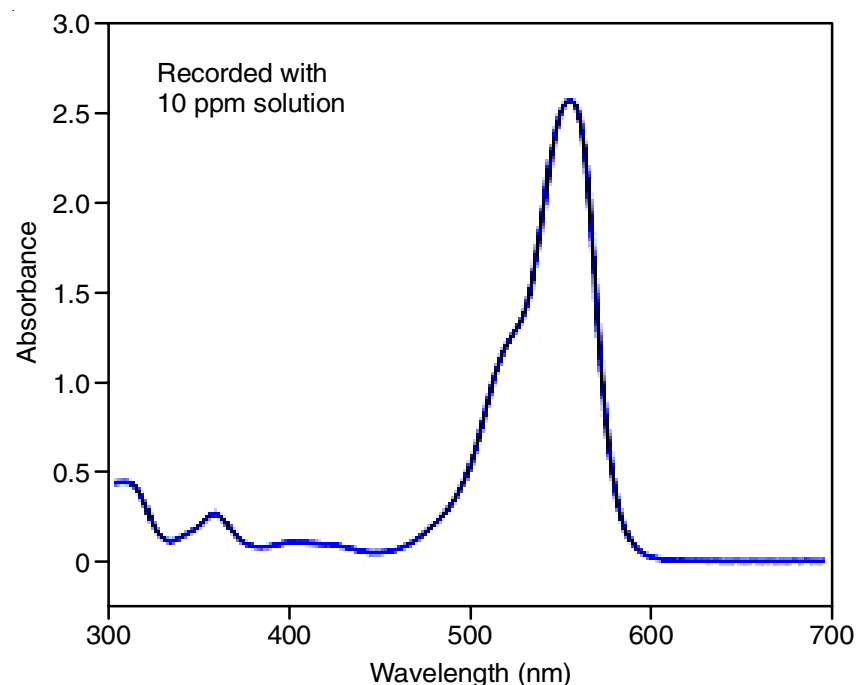

Fig. 8. UV-visible spectra of rhodamine-B solution (10 ppm)

Effect of initial dye concentration: The effect of initial dye concentration was studied by varying the concentration of rhodamine B dye solution from $2 \mathrm{ppm}$ to $16 \mathrm{ppm}$ and by keeping the weight of $\mathrm{x} \%$ GO-nano titania materials $(10 \mathrm{mg} /$ $100 \mathrm{~mL}$ ) constant. The degradation efficiency was found to increase with increase in the concentration of the dye solution from 2 to $10 \mathrm{ppm}$. However, the degradation tendency was decreased with further increase in the concentration of the dye. It may be due to decrease in the active sites on the surface of the composite material (for a fixed weight) with increase in the dye molecules. Increased concentrations also give intense colour to the dye solution and decrease the photon count on the surface of the material. This condition limits the production of hydroxyl and superoxide radicals on its surface. At still higher concentrations, the photocatalytic degradation was almost negligible. From Fig. 9, it was clear that the maximum photocatalytic degradation can be observed for $10 \mathrm{ppm}$ concentration of rhodamine B solution.

Effect of graphene oxide composition on degradation efficiency: In the visible region, the degradation efficiency of GO is $75 \%$ and $1-2 \%$ (approx.) for the nano titania particles [10]. Hence, the introduction of GO on the surface of nano 


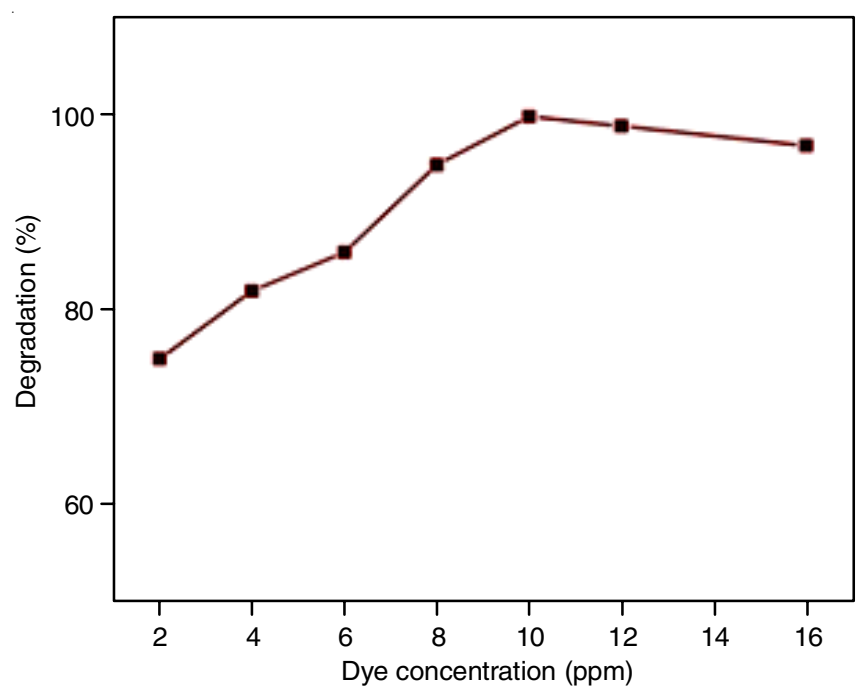

Fig. 9. Effect of initial dye concentration of rhodamine B, catalyst load = $10 \mathrm{mg}$ (fixed weight)

titania particles increased the adsorption capacity and enhanced the photocatalytic degradation of dye in the visible region. In the absence of light, almost there was no photocatalytic degradation and it signifies that the degradation by light may be ignored. The efficiencies of $x \%$ GO-nano titania composites in the degradation of rhodamine $\mathrm{B}$ dye $(10 \mathrm{ppm})$ were significantly improved from 62 to $100 \%$ with increase in the content of graphene oxide from 1 to $10 \%$ in the composite materials. Among the synthesized composite materials, $10 \%$ GO-nano titania composite has shown almost $100 \%$ degradation and in the present work, it may be considered as a fine material in removing the organic pollutants in wastewater under visible light irradiation. On further increasing the wt $\%$ of GO in composites, there was no appreciable change in the degradation efficiency of dye. It may be due to the agglomeration of GO particles in $15 \%$ GO- nano titania material thereby decreasing the specific surface areas of GO-nano titania composites and results were shown in Fig. 10.

Effect of amount of $10 \%$ GO-nano titania composite on photocatalytic degradation of rhodamine $B$ dye: The

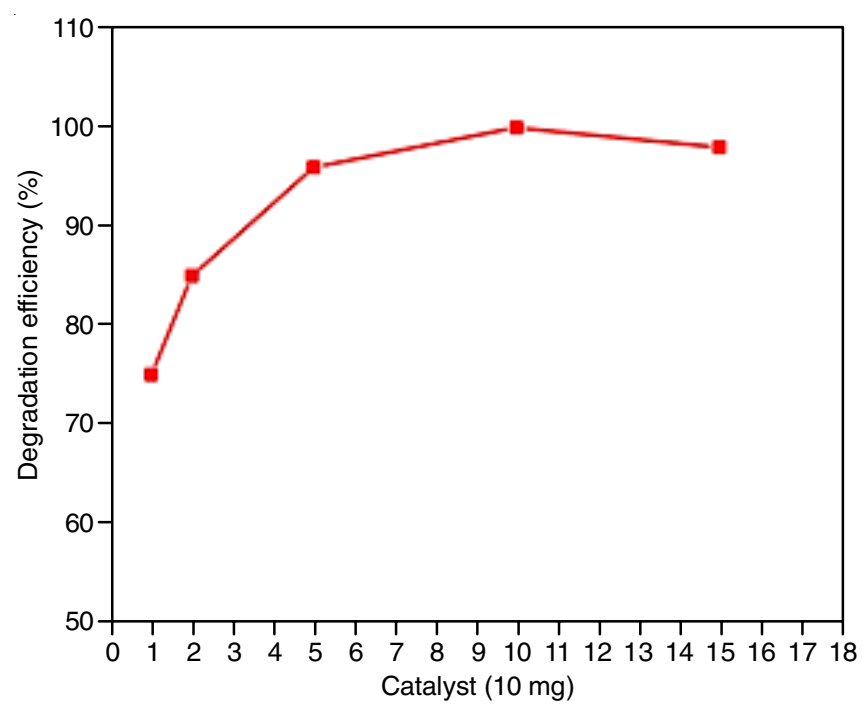

Fig. 10. Effect of $\%$ graphene oxide composition in composites on the degradation efficiency of rhodamine $\mathrm{B}$ under visible light irradiation, catalyst load $=10 \mathrm{mg}$, $[$ Dye $]=10 \mathrm{ppm}$ effect of photocatalyst load on the photocatalytic degradation of rhodamine B was examined with $10 \%$ GO-nano titania material. The dosage amount varied from 2 to $50 \mathrm{mg} / 100 \mathrm{~mL}$ of dye solution and all the observations were carried out at room temperature. It was observed that with the increase of catalyst load from 2 to $10 \mathrm{mg} / 100 \mathrm{~mL}$ of dye solution, the degradation efficiencies were appreciably improved. Further increase of the catalyst load from 10 to $50 \mathrm{mg} / 100 \mathrm{~mL}$ decreased the degradation efficiency of dye (Fig. 11). This phenomenon may be because of the increase in the amount of catalyst dosage, which would increase the reactive sites that can correspondingly produce more reactive oxidative species. However, too much catalyst dispersed in the system will possibly increase light scattering and decrease light penetration, resulting in the reduction of degradation efficiency of photocatalyst [19]. Hence, it was optimized that $10 \%$ GO-nano titania composite material with $10 \mathrm{mg}$ load/100 mL was an appreciable amount of photocatalyst for degradation of $10 \mathrm{ppm}$ dye solution.

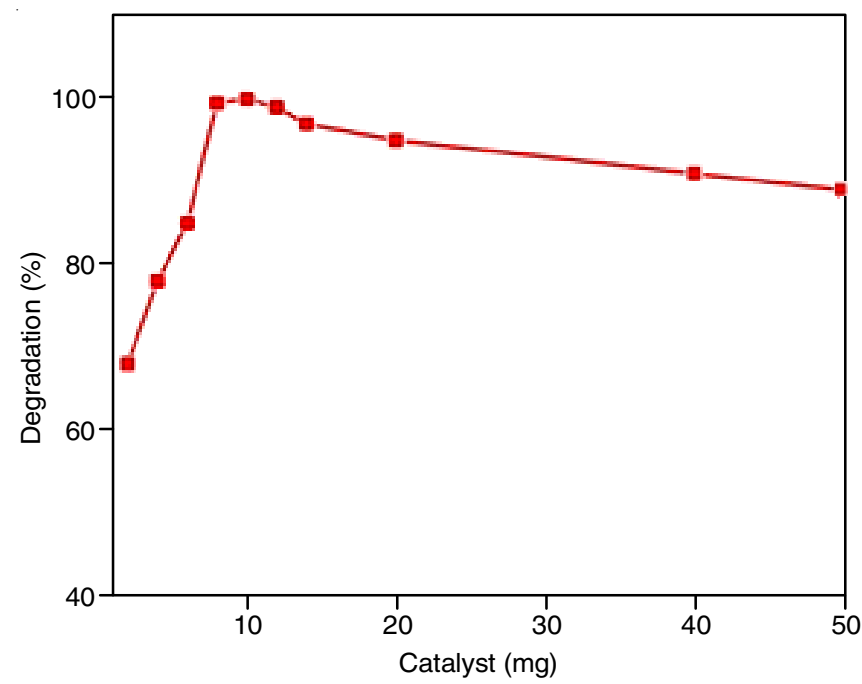

Fig. 11. Effect of amount of 10\% GO-nano titania composite on visible light induced photocatalytic degradation of rhodamine B dye, [Dye] $=10 \mathrm{ppm}$

Effect of time of irradiation on photocatalytic degradation: The effect of irradiation time on visible light induced photocatalytic degradation of rhodamine B dye was investigated in the presence of composite materials. It was observed that time of irradiation was decreased from 180 to $60 \mathrm{~min}$ with increased graphene oxide weight in $\mathrm{x} \% \mathrm{GO}$-nano titania composite materials (Fig. 12). $100 \%$ photocatalytic degradation was achieved with $10 \mathrm{mg}$ of $10 \%$ GO-nano titania composite material in $60 \mathrm{~min}$. This may be due to enhanced absorption of dye molecules with increased GO content and increased photocatalytic performance of the photocatalysts. There was no significant change in the photocatalytic degradation with 15\% GO-nano titania material compared with $10 \%$ GO-nano titania material. This condition may be due to agglomeration of high content GO particles in 15\% GO-nano titania material and decreasing the active sites on the surface of composite.

Effect of $\mathbf{p H}$ of initial dye solution: The effect of $\mathrm{pH}$ on $10 \mathrm{ppm}$ rhodamine $\mathrm{B}$ dye solution for photocatalytic degradation under visible light was studied by varying the concentrations of $\mathrm{HNO}_{3}$ and $\mathrm{NaOH}$ and using $10 \%$ GO-nano titania material 


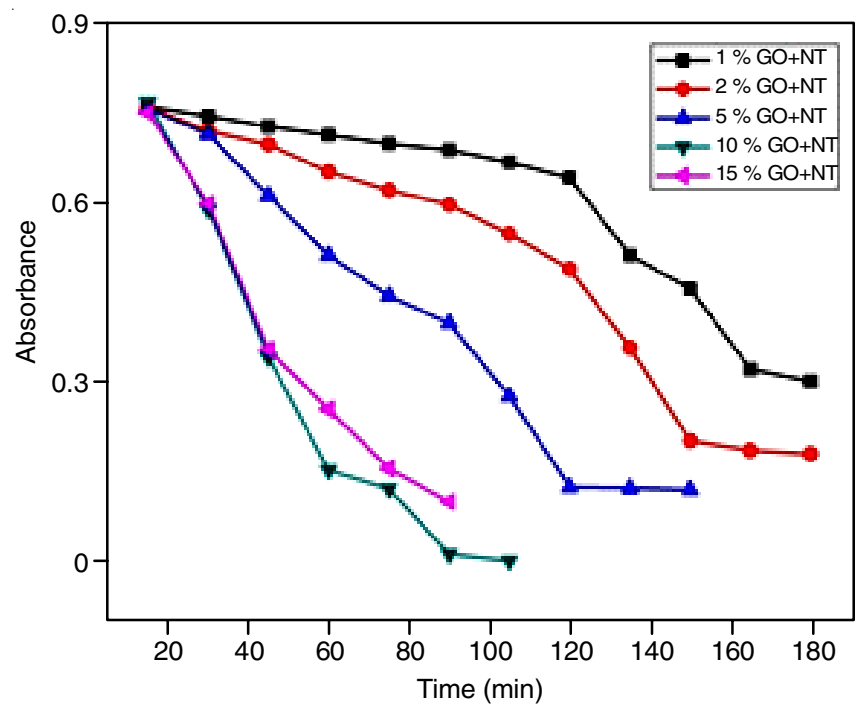

Fig. 12. Effect of time of irradiation on photocatalytic degradation of rhodamine $\mathrm{B}$ under visible light in the presence of $\mathrm{x} \% \mathrm{GO}$-nano titania composites, catalyst load $=10 \mathrm{mg}$, [Dye] $=10 \mathrm{ppm}$

(Fig. 13). These effects were studied by varying $\mathrm{pH}$ of dye solution from 1.5 to 14.0. A slow decrease in degradation efficiency was observed as the $\mathrm{pH}$ increased and reached a minimum at around $\mathrm{pH}$ 8.0. With further increase in $\mathrm{pH}$, the degradation efficiency slowly increased and reached a maximum in the alkaline range. In the acidic range, perhydroxyl radical can form hydrogen peroxide giving rise to hydroxyl radical and in alkaline condition, it could be attributed to increase of hydroxyl ions, which induces more hydroxyl radical formation [20]. These hydroxyl radicals would oxidize rhodamine $\mathrm{B}$ dye molecules. Hence, at extremely acidic/alkaline $\mathrm{pH}$ range superior degradation efficiency was noted and the results are in close agreement with the reported work [16].

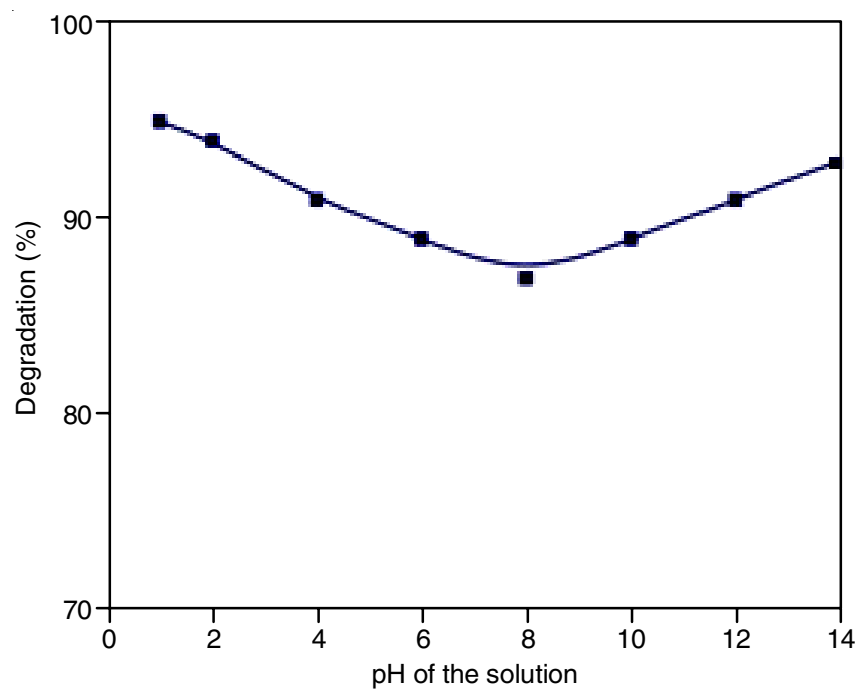

Fig. 13. Effect of $\mathrm{pH}$ on initial dye solution; catalyst $=10 \%$ GO- nano titania, catalyst load $=10 \mathrm{mg}$, [Dye $]=10 \mathrm{ppm}$

Effect of temperature: The effect of temperature on the apparent photocatalytic degradation of $10 \mathrm{ppm}$ rhodamine-B dye solution is shown in Fig. 14. The temperature of the reacting system was varied from 20 to $80{ }^{\circ} \mathrm{C}$ to identify the effect of photocatalytic performance of the dye solutions under visible

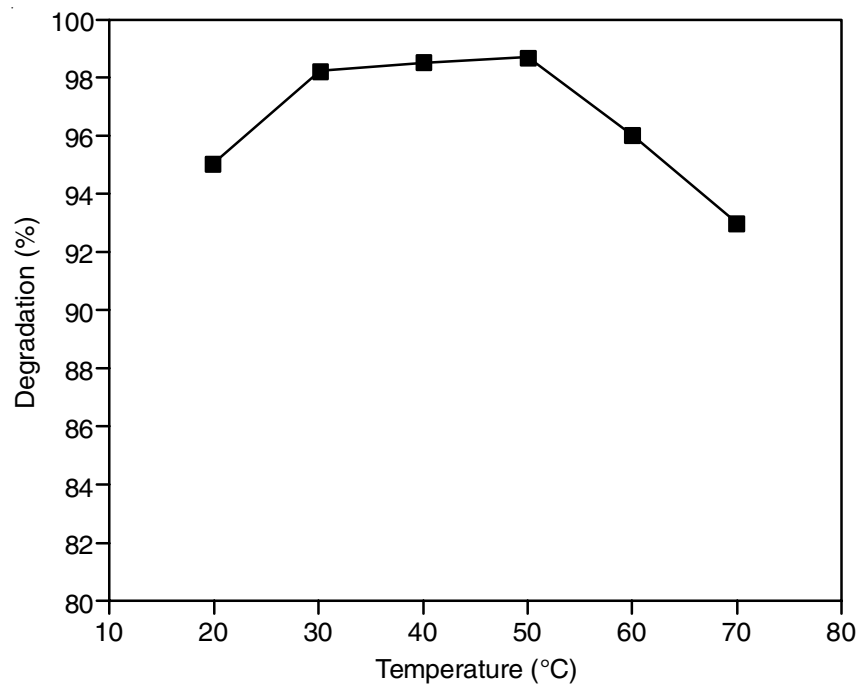

Fig. 14. Effect of temperature on visible light induced photocatalytic degradation of rhodamine $\mathrm{B}$; catalyst $=10 \%$ GO-nano titania, catalyst load $=10 \mathrm{mg},[$ Dye $]=10 \mathrm{ppm}$

light irradiation. It was observed that photocatalytic performance was better in the range of 30 to $50^{\circ} \mathrm{C}$ and are almost similar to the reported results [21]. A poor photocatalytic performance was observed at extremely low and high temperatures. It may be due to decrease in mass transfer of pollutants to the surface of photocatalysts at low temperature and recombination of charge carriers and desorption of adsorbed dye molecules on the photocatalysts at high temperature.

Plausible photocatalytic mechanism: The major reaction steps, the routes of formation of radicals and the photocatalytic degradation of rhodamine-B dye is described in Scheme-I. On visible light irradiation, the electrons present in valence band (VB) of nano titania particles gets excited to conduction band (CB). This stage is referred to as semiconductor's photoexcitation stage creating a negative-electron $\left(\mathrm{e}^{-}\right)$and positivehole $\left(\mathrm{h}^{+}\right)$pair. In the absence of $\mathrm{GO}$, these $\mathrm{e}^{-} / \mathrm{h}^{+}$pairs combine vigorously and results in a very low photocatalytic activity. The $d$-orbital (conduction band) of nano titania and $\pi$-orbital of graphene oxide interact and forms a d- $\pi$ overlap [22]. The nano titania particles absorbs the visible light and transports the excited state electron from its conduction band into $\pi$-orbital of GO and the excited electrons can be shuttled easily along the conducting network of GO surface thereby decreasing $\mathrm{e}^{-} /$ $\mathrm{h}^{+}$recombination in the nano titania particles. This negative electron subsequently transfers to the surface of composite material to react with water and oxygen and yields hydroxyl radicals and super oxide ions, respectively. These hydroxyl radicals and super oxide ions would oxidize rhodamine B dye.

$$
\begin{aligned}
& \mathrm{TiO}_{2}+\mathrm{h} \nu \longrightarrow\left(\mathrm{e}_{\mathrm{CB}}^{-}\right)+\left(\mathrm{h}_{\mathrm{VB}}^{+}\right) \longrightarrow \mathrm{GO}^{-}\left(\mathrm{e}^{-}\right)+\mathrm{h}^{+} \\
& \mathrm{TiO}_{2}\left(\mathrm{e}^{-}\right)+\mathrm{O}_{2} \longrightarrow \mathrm{O}_{2}^{-}+\mathrm{TiO}_{2} \longrightarrow \mathrm{H}_{2} \mathrm{O} \\
& \mathrm{TiO}_{2}\left(\mathrm{~h}_{\mathrm{VB}}^{+}\right)+\mathrm{OH}^{-}+\mathrm{OHO}_{2} \longrightarrow \mathrm{OH}^{\cdot}+\mathrm{TiO}_{2} \\
& \mathrm{GO}\left(\mathrm{e}^{-}\right)+\mathrm{O}_{2} \longrightarrow \mathrm{O}_{2}^{\cdot}+\mathrm{GO} \stackrel{\mathrm{H}_{2} \mathrm{o}^{\cdot}}{\longrightarrow} \mathrm{OH}+\mathrm{GO} \\
& \mathrm{OH}^{\cdot}+\text { Rhodamine } \mathrm{B} \longrightarrow \text { Degraded product } \\
& \mathrm{O}_{2}^{--}+\text {Rhodamine } \mathrm{B} \longrightarrow \text { Degraded product }
\end{aligned}
$$

Scheme-I: Photocatalytic degradation reactions of rhodamine-B in presence of $\mathrm{x} \%$ GO-nano titania composites under visible light irradiation 


\section{Conclusion}

A one step organic solvent free controlled hydrolysis of $\mathrm{TiCl}_{4}$ in water (ratio of 1:50 mL) medium and in situ dispersion of graphene oxide solution to synthesize of graphene oxidenano titania composite materials was reported and their photocatalytic activity on the degradation of rhodamine $B$ was evaluated under visible light. The characterization techniques XRD, FESEM, FTIR, HRTEM and UV-Vis DRS illustrated the nature and structure of the prepared nanocomposite materials. It was optimized that rhodamine B dye solution of $10 \mathrm{ppm}$ concentration was $100 \%$ degraded in the presence of $10 \%$ GO-nano titania composite material with $10 \mathrm{mg}$ catalyst load at extremely acidic or alkaline $\mathrm{pH}$ range around $30-50{ }^{\circ} \mathrm{C}$ under visible light irradiation. Further, the time of irradiation was reduced from 180 to 60 min with $10 \%$ GO-nano titania composite material.

\section{ACKNOWLEDGEMENTS}

The present work was funded by University Grants Commission SERO-Hyderabad, India through minor research project (F.No. 6128/15 to SSR). The authors thank Advanced Analytical Lab, Andhra Univeristy for FE-SEM, FTIR analysis, Centre for Advanced Instrumentation, NIT-Warangal, India for XRD and UV-Vis DRS analysis \& Sophisticated Analytical Instrumentation Facility (SAIF), Cochin university, Cochin, India for HRTEM analysis.

\section{REFERENCES}

1. D. Zhao, G. Sheng, C. Chen and X. Wang, Appl. Catal. B, 111-112, 303 (2012);

https://doi.org/10.1016/j.apcatb.2011.10.012.

2. R.F. Howe, Dev. Chem. Eng. Miner. Process., 6, 55 (1998); https://doi.org/10.1002/apj.5500060105.

3. S.T. Aruna and K.C. Patil, J. Mater. Synth. Process., 4, 175 (1996).

4. D.W. Bahnemann, Isr. J. Chem., 33, 115 (1993); https://doi.org/10.1002/ijch.199300017.

5. T.D. Nguyen-Phan, V.H. Pham, E.W. Shin, H.-D. Pham, S. Kim, J.S. Chung, E.J. Kim and S.H. Hur, Chem. Eng. J., 170, 226 (2011); https://doi.org/10.1016/j.cej.2011.03.060.
6. M.M. Mahlambi, C.J. Ngila and B.B. Mamba, J. Nanomater, Article ID 790173 (2015); https://doi.org/10.1155/2015/790173.

7. T.N. Murthy, P. Suresh, A.M. Umabala and A.V.P. Rao, Int. J. Recent Scientific Res., 7, 10895 (2016).

8. K. Zhou, Y. Zhu, X. Yang, X. Jiang and C. Li, New J. Chem., 35, 353 (2010); https://doi.org/10.1039/C0NJ00623H.

9. S. Stankovich, D.A. Dikin, R.D. Piner, K.A. Kohlhaas, A. Kleinhammes, Y. Jia, Y. Wu, S.B.T. Nguyen and R.S. Ruoff, Carbon, 45, 1558 (2007); https://doi.org/10.1016/j.carbon.2007.02.034.

10. X. Meng, L. Jiang, W. Wang and Z. Zhang, Int. J. Photoenergy, Article ID 747024 (2015); https://doi.org/10.1155/2015/747024.

11. M. Addamo, V. Augugliaro, A. Di Paola, E. García-López, V. Loddo, G. Marcì, R. Molinari, L. Palmisano and M. Schiavello, J. Phys. Chem., 108, 3303 (2004); https://doi.org/10.1021/jp0312924.

12. M. Addamo, V. Augugliaro, A. Di Paola, E. García-López, V. Loddo, G. Marcì and L. Palmisano, Colloids Surf. A, 265, 23 (2005); https://doi.org/10.1016/j.colsurfa.2004.11.048.

13. G. Jiang, Z. Lin, C. Chen, L. Zhu, Q. Chang, N. Wang, W. Wei and H. Tang, Carbon, 49, 2693 (2011); https://doi.org/10.1016/j.carbon.2011.02.059.

14. G. Williams, B. Seger and P.V. Kamat, ACS Nano, 2, 1487 (2008); https://doi.org/10.1021/nn800251f.

15. E.R. Nestmann, G.R. Douglas, T.I. Matula, C.E. Grant and D.J. Kowbel, Cancer Res., 39, 4412 (1979).

16. K. Shen and M.A. Gondal, J. Saudi Chem. Soc., 21, S120 (2017); https://doi.org/10.1016/j.jscs.2013.11.005.

17. H. Zhang, X. Lv, Y. Li, Y. Wang and J. Li, ACS Nano, 4, 380 (2010); https://doi.org/10.1021/nn901221k.

18. B. Paulchamy, G. Arthi and B.D. Lignesh, J. Nanomed. Nanotechnol., 6, 253 (2015); https://doi.org/10.4172/2157-7439.1000253.

19. K. Byrappa, A.K. Subramani, S. Ananda, K.M.L. Rai, R. Dinesh and M. Yoshimura, Bull. Mater. Sci., 29, 433 (2006); https://doi.org/10.1007/BF02914073.

20. E.T. Soares, M.A. Lansarin and C.C. Moro, Braz. J. Chem. Eng., 24, 354 (2007); https://doi.org/10.1590/S0104-66322007000100003.

21. H.J. Huang, Y.S. Zhen, P.-Y. Li, S.-D. Tzeng and H.-P. Chiang, Opt. Express, 24, 15603 (2016); https://doi.org/10.1364/OE.24.015603.

22. W.-K. Jo and H.-J. Kang, Powder Technol., 250, 115 (2013); https://doi.org/10.1016/j.powtec.2013.10.017. 